\title{
Simulation tridimensionnelle de la blocométrie naturelle de massifs rocheux
}

\section{Three dimensional simulation of block size distribution of rock masses}

\author{
J. XU, R. COJEAN, M. ARNOULD \\ CGI, Ecole des Mines de Paris"
}

Rev. Franç: Géotech. n58, pp. 31-40 (janvier 1992)

\section{Résumé}

Cet article présente un modèle général de simulation à trois dimensions de la blocométrie naturelle des massifs rocheux. Il est constitué de quatre parties : 1. analyse statistique et simulation à trois dimensions des discontinuités ; 2. analyse de la connectivité des discontinuités simulées; 3 . identification des blocs discrets intersectés par des discontinuités connectées ; 4. caractérisation de la blocométrie de massifs rocheux. Trois types de représentation (distribution de taille, distribution de taille pondérée, distribution de l'orientation des blocs) sont utilisés. Les résultats des exemples réels sont donnés dans les sections correspondantes de l'article.

\footnotetext{
Abstract

This paper presents a general model for simulating three dimensional natural rock mass granulometry. It consists of four parts : 1 . statistic analysis and three dimensional simulation of rock mass fractures ; 2 . connectivity study of simulated fractures ; 3 . identification of distinct blocks intersected by the fractures: 4. characterization of rock mass granulometry. Three representative methods, such as size distribution, weighted size distribution and orientation distribution of blocks, are used. The results of some real examples are given in the correspondent sections of the paper.
} 


\section{INTRODUCTION}

Les développements récents dans l'analyse de la stabilité et dans l'évaluation de la fragmentation des massifs rocheux par explosif exigent une évaluation initiale de la blocométrie naturelle de ceux-ci. Depuis le travail original de CUNDALL (1972), la méthode des éléments discrets s'est développée pour étudier le comportement mécaniques des milieux fissurés (CUNDALL, 1988). Cette méthode considère un massif rocheux comme un système de blocs discrets délimités par des discontinuités, et donc elle exige un système de blocs pré-défini. La fragmentation d'un massif rocheux par explosif est contrôlée pour partie par sa blocométrie initiale (distributions de taille et d'orientation des blocs). Un modèle de la blocométrie naturelle nous permet de faire l'étude comparative entre celle-ci et la granulométrie du tas abattu pour optimiser l'utilisation de l'énergie explosive.

Certains programmes de simulation des discontinuités à trois dimensions ont été développés pour lanalyse d'écoulements souterrains. LONG et al., (1985) ont réalisé un modèle de système de discontinuités à trois dimensions. ANDERSSON et DVERSTORP (1987) ont développé un modèle de simulation conditionnelle de réseaux de discontinuités discrètes. Mais l'application de sustèmes de discontinuités simulées à l'analyse mécanique ou à l'évaluation de la blocométrie d'un massif rocheux rencontre la difficulté d'identification des blocs discrets séparés par des discontinuités.

LIN et al. (1987) ont proposé une méthode géométrique pour identifier le système de blocs. Dans cette méthode, un bloc (polyèdre) est considéré comme un complexe orienté dont quelques propriétés topologiques peuvent être utilisées comme critères pour identifier les blocs. Cette méthode peut considérer, pour la première fois, les blocs convexes ou concaves.

Le générateur de blocs RESOBLOC développé par HELIOT (1988) considère l'histoire géologique de développement de différentes familles de fissures pour construire une base de données de blocs d'un massif. Ce modèle est basé sur la génération de différentes familles de fissures. Chaque nouvelle famille de fissures est introduite à l'intérieur de domaines délimités par les fissures déjà générées. La méthode de simulation de fissures diffère des modèles normalement utilisés et le modèle peut être considéré comme un modèle hiérarchique. Il donne une bonne représentation de certains massifs rocheux, par exemple, un massif rocheux sédimentaire.

Dans cet article, nous présentons la méthode utilisée dans le programme SIMBLOC de simulation tridimensionnelle de la blocométrie naturelle des massifs rocheux. A partir de la configuration des discontinuités simulées, une étude de la connectivité des discontinuités est faite d'abord pour éliminer les discontinuités non-connectées et fournir un modèle géométrique pour l'analyse d'écoulements de fluides et de la blocométrie. Des propriétés topologiques d'un polyèdre sont utilisées pour identifier un bloc discret. Trois méthodes de caractérisation de la blocométrie d'un massif rocheux sont présentées : distribution de taille, distribution de taille pondérée et distribution de l'orientation des blocs.

\section{SIMULATION TRIDIMENSIONNELLE DE DISCONTINUITÉS}

La méthode de simulation présentée dans cet article est une méthode généralement utilisée par d'autres auteurs comme BAECHER (1983), LONG et al. (1985), ANDERSSON et DVERSTORP (1987). Une discontinuité est modélisée comme un disque de rayon $r$ avec une orientation $\alpha$ et un pendage $\beta$. Ces paramètres géométriques sont aléatoires et suivent certaines lois de distributions pour chaque famille de discontinuités.

L'orientation et le pendage $(\alpha, \beta)$ d'une discontinuité peuvent être considérés comme les coordonnées sphériques de la normale à la discontinuité et ils sont habituellement considérés indépendants. Si nous considérons le vecteur d'une normale comme un point sur un hémisphère unitaire, alors la distribution de ce vecteur peut être décrite par la distribution normale hémisphérique (MAHTAB et al., 1972)

$\mathrm{P}(\theta)=(1-\exp [\mathrm{k}(\cos \theta-1)]) /[1-\exp (-\mathrm{k})]$

avec $\mathrm{k}$ paramètre de dispersion ;

$\theta$ angle par rapport à la direction moyenne d'une famille

$\mathrm{P}(\theta)$ probabilité que la normale d'une discontinuité fasse un angle inférieur ou égal à $\theta$ par rapport à la normale ou pôle de la famille.

L'estimation des paramètres à trois dimensions comme l'extension (rayon de disque) et la densité des discontinuités se base sur des relations probabilistes appliquées aux paramètres mesurables à deux dimensions comme l'extension (longueur) et la densité des traces des discontinuités sur un affleurement.

La distribution de l'extension peut être estimée par les données de la longueur de traces d'intersection entre une famille de discontinuités et la surface de mesure. WARBURTON (1980) a étudié la relation probabiliste entre le paramètre de distribution du rayon $r$ et celui de distribution de la longueur de trace 1 pour différentes méthodes de mesure (fenêtre de mesure et ligne de mesure). Pour la reconnaissance en fenêtre, la relation s'écrit :

$$
\mathrm{E}(\mathrm{l})=\frac{\pi}{4} \frac{\mathrm{E}\left(\mathrm{r}^{2}\right)}{\mathrm{E}(\mathrm{r})}=\frac{\pi}{4} \frac{\sigma^{2}+\mu^{2}}{\mu}
$$

avec $E(r), E\left(r^{2}\right)$ deux premiers moments centrés de $r$ $\mu, \sigma$ moyenne et écart-type de $r$

E(l) espérance mathématique de 1 .

L'évaluation de cette équation exige une distribution supposée de $r$. En pratique, nous supposons que le rayon $\mathrm{r}$ suit la même loi que la longueur de trace 1 . Les études expérimentales montrent que 1 suit habi- 
tuellement la loi log-normale ou exponentielle (BAECHER, 1983) dont l'estimation des paramètres est bien étudiée et peut être réalisée par des procédés traditionnels (PRIEST et HUDSON, 1981 ; PAHL, 1981).

La densité volumique $\lambda$ d'une famille de discontinuités est définie comme le nombre de discontinuités par unité de volume dans un massif rocheux et la densité de surface $\lambda$ ' est définie comme le nombre de traces d'intersection par unité de surface. Des relations mutuelles entre $\lambda$ et $\lambda^{\prime}$ sont déduites par WARBURTON (1980) pour différents modèles de formes de discontinuité. Pour le modèle de disque, cette relation s'écrit :

$$
\lambda=\lambda^{\prime} /(2 \cdot \sin \alpha \cdot E(r))
$$

avec $\alpha$ angle entre la normale à une famille de discontinuités et la normale à la surface de mesure. Un programme est développé spécialement pour le traitement statistique de données acquises à deux dimensions. Dans ce programme, la méthode de la classification automatique est utilisée pour déterminer les familles directionnelles des discontinuités, et pour chaque famille, certains paramètres statistiques comme les moyennes et les écarts-types de l'extension, de l'orientation, du pendage et de la densité sont calculés simultanément. Les résultats d'un exemple réel de 183 discontinuités mesurées sur la paroi d'un tunnel de reconnaissance dans un massif de granite sont montrés dans le tableau 1. La figure 1 est la projection stéréographique des discontinuités sur laquelle on peut visualiser approximativement trois familles.

Dès que les paramètres statistiques de la géométrie des discontinuités sont obtenus, la simulation peut être réalisée d'une manière standard suivant la loi de distribution de chaque paramètre. Dans le programme, le domaine de simulation est dilaté par rapport au

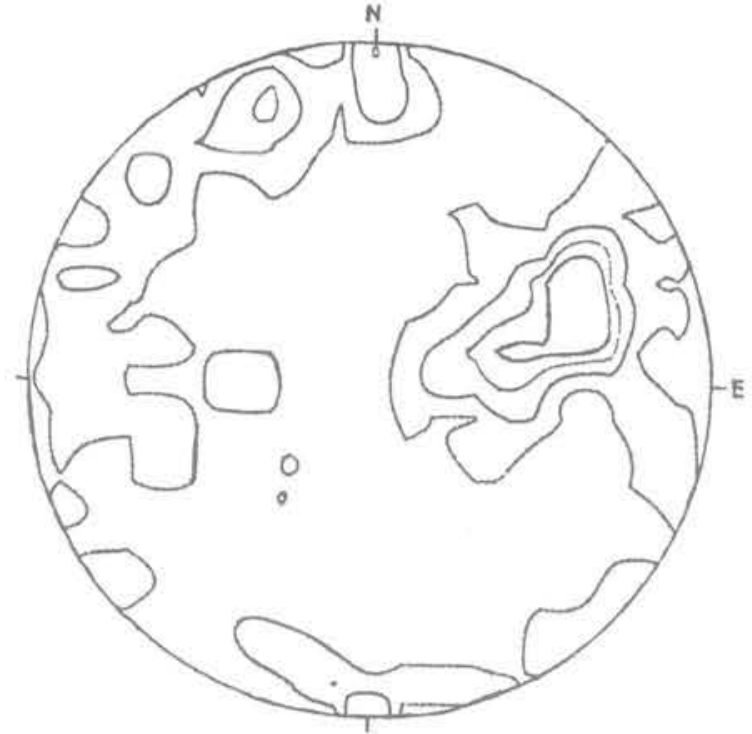

Fig. 1. - Projection stéréographique des discontinuités.

Fig. 1. - Sterographic projection of discontinuities.

domaine réel suivant la valeur de l'extension des discontinuités pour générer une marge tampon et diminuer ainsi l'influence de marge. Un exemple de configuration de discontinuités simulées dans un talus suivant les données des cinq familles du tableau 1 est montré dans la figure 2.

\section{ETUDE DE LA CONNECTIVITÉ DE DISCONTINUITÉS}

Une discontinuité peut constituer un cheminement d'écoulement ou une facette d'un bloc à condition qu'elle soit connectée avec le réseau global des dis-

Tableau 1. - Résultats d'analyse statistique des données de discontinuités.

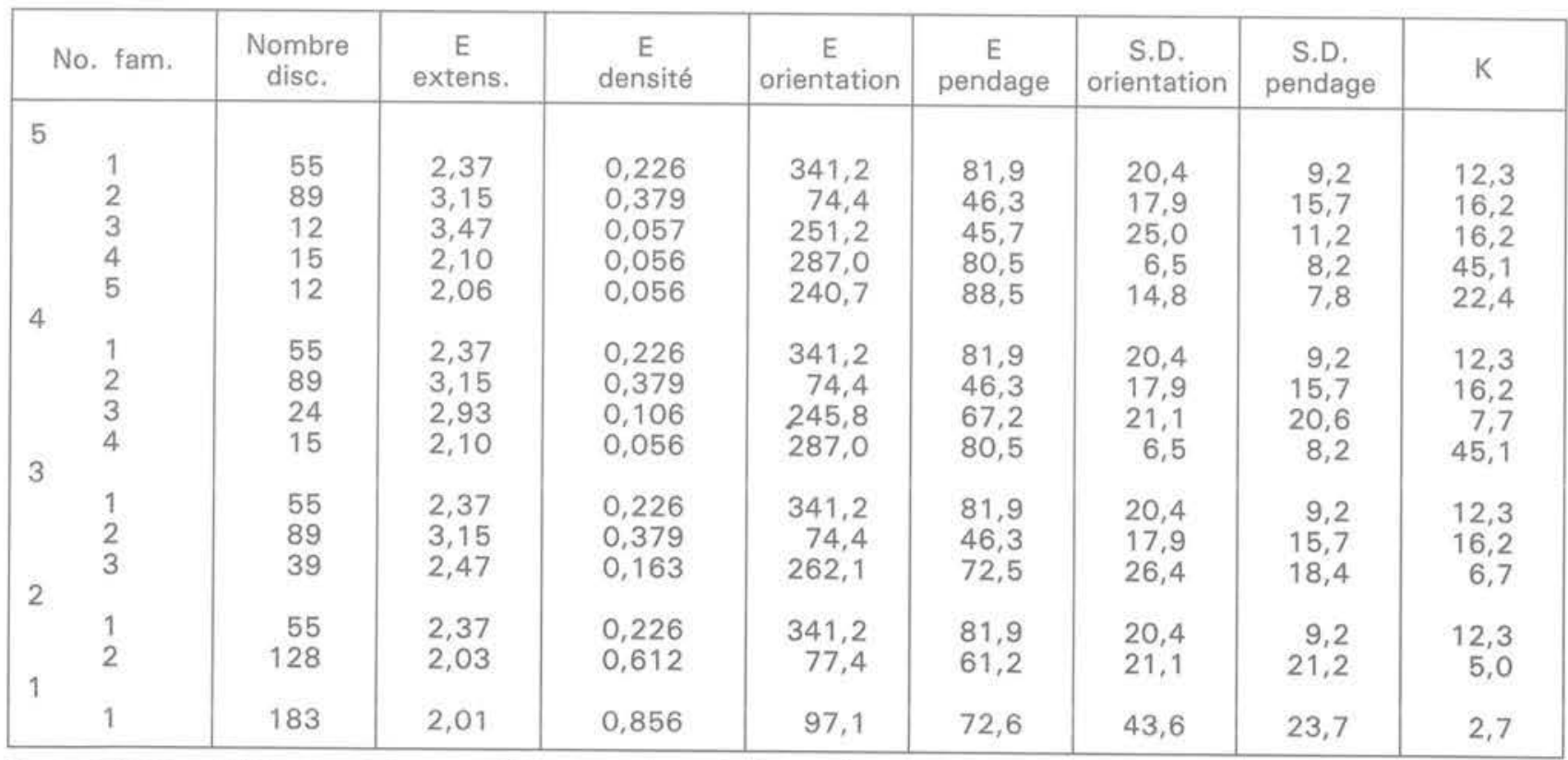

E : moyenne - S.D. : écart-type ; - K : paramètre de dispersion. 


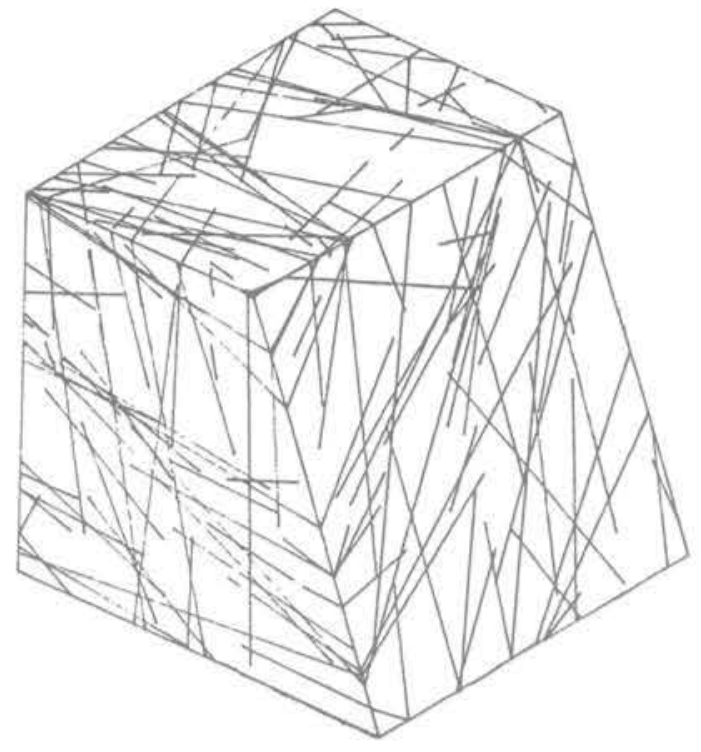

Fig. 2. - Configuration des discontinuités simulées.

Fig. 2. - Simulated discontinuities.

continuités. Par exemple, pour un problème de blocométrie, elle doit intersecter au moins trois autres discontinuités ou les limites du domaine et les traces des intersections doivent se connecter mutuellement sur la discontinuité. Donc l'étude de la connectivité se base sur l'identification des intersections entre les discontinuités ou les limites du domaine.

L'intersection entre le disque d'une discontinuité $\mathrm{Fi}$ et le disque d'une discontinuité Fj peut être identifiée par les trois étapes suivantes : 1 . déterminer la ligne d'intersection entre deux plans contenant $\mathrm{Fi}$ et $\mathrm{Fj} ; 2$. calculer la distance entre la ligne d'intersection et les centres de $\mathrm{Fi}$ et $\mathrm{Fj}$. Si la condition de l'extension n'est pas satisfaite, les deux discontinuités ne s'intersectent pas ; 3 . déterminer la partie commune d'intersection de $\mathrm{Fi}$ et $\mathrm{Fj}$. Si elles ne sont pas décalées, il existe une partie commune et les deux discontinuités s'intersectent. Les limites du domaine de simulation peuvent être interprétées comme des polygones convexes, leurs intersections avec des discontinuités peuvent être identifiées par une méthode semblable à celle présentée ci-dessus. Quand cette procédure est appliquée à toutes les discontinuités dont l'ensemble est noté par $\mathbb{F}$, alors toutes les intersections auront été identifiées et l'ensemble d'intersections est noté par II.

En effet, un système de discontinuité et ses relations d'intersections peuvent être décrits par un graphe, si nous considérons une discontinuité comme un sommet, et une intersection comme une arête du graphe. Cela s'applique à un système à trois dimensions ainsi qu'à deux dimensions. La figure 3 montre un exemple simplifié bidimensionnel.

Le degré de connectivité d'un système de discontinuités peut être défini comme le nombre minimum d'intersections qu'une discontinuité possède dans le système. Dans la figure 2 , il existe certaines discontinuités isolées qui n'intersectent aucune autre, le degré de connectivité du système est donc zéro. Un système de faible degré de connectivité peut devenir un système de degré plus fort par l'élimination des discontinuités qui ne possèdent pas assez d'intersections afin d'établir des modèles géométriques pour certaines applications pratiques (écoulement des fluides, blocométrie). Si nous notons l'ensemble de discontinuités intersectant la discontinuité $\mathrm{Fi}$ par $\Gamma(\mathrm{Fi})$ et l'ensemble d'intersections sur $\mathrm{Fi}$ par $\mathrm{T}(\mathrm{Ii})$, alors pour le degré $\mathrm{n}$, l'algorithme d'élimination d'une discontinuité $\mathrm{Fi}$ intersectant moins que $n$ autres et des intersections associées à $\mathrm{Fi}$ s'écrit :

$$
\begin{aligned}
& \mathbb{F}-\mathbb{F}-F i \\
& \mathbb{I}-\mathbb{I}-\Gamma(\mathrm{Ii}) \\
& \Gamma(\mathrm{Fj})-\Gamma(\mathrm{Fj})-\mathrm{Fi}, \mathrm{Fj} \in \Gamma(\mathrm{Fi}) \\
& \Gamma(\mathrm{Fi})-\phi \\
& \Gamma(\mathrm{Ij})-\Gamma(\mathrm{lj})-\mathrm{ik}, \mathrm{ik} \in \Gamma(\mathrm{li}) \cap \Gamma(\mathrm{Ij}) \\
& \Gamma(\mathrm{Ii})-\phi
\end{aligned}
$$

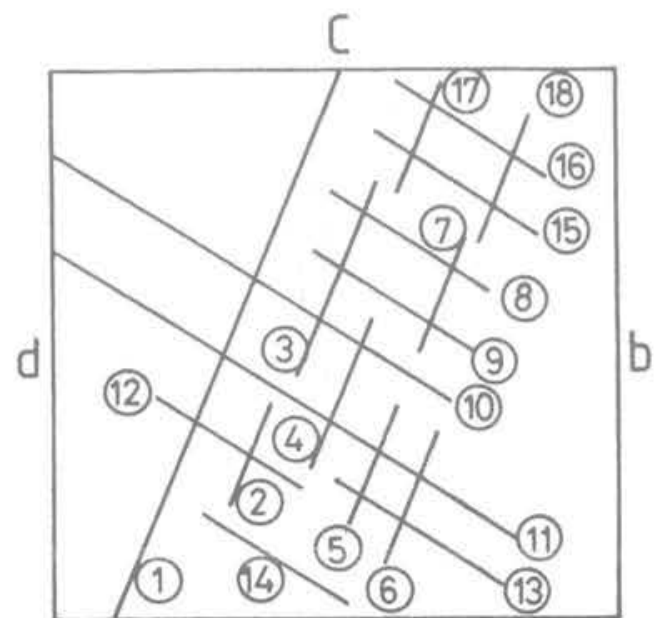

a

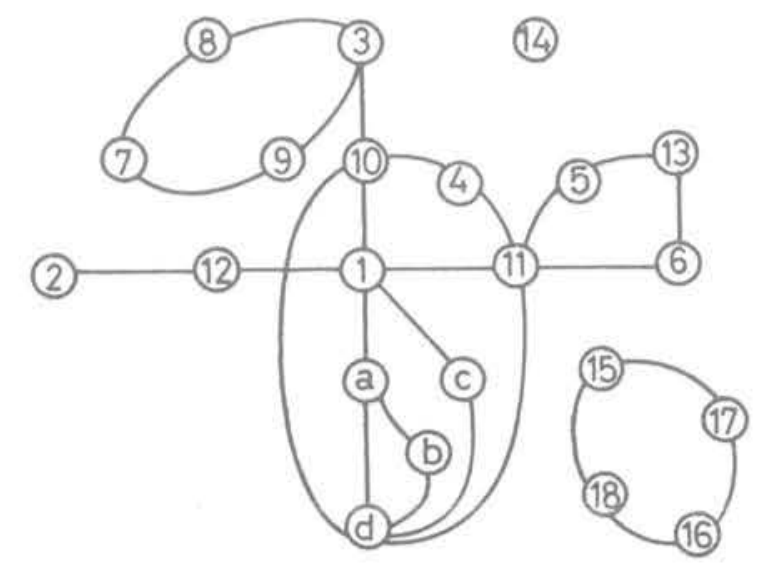

B
A

Fig. 3. - A. Un exemple de système de discontinuités à deux dimensions. B. Représentation en graphe des relations d'intersection.

Fig. 3. - A. An example of two dimensional discontinuity system. B. Graph representation of intersection relationships. 
L'algorithme (4) doit être appliqué à toutes les discontinuités du système et répété jusqu'à ce que chaque discontinuité possède au moins $n$ intersections. La figure 4 montre le résultat de degré 2 du système dans la figure 2 .

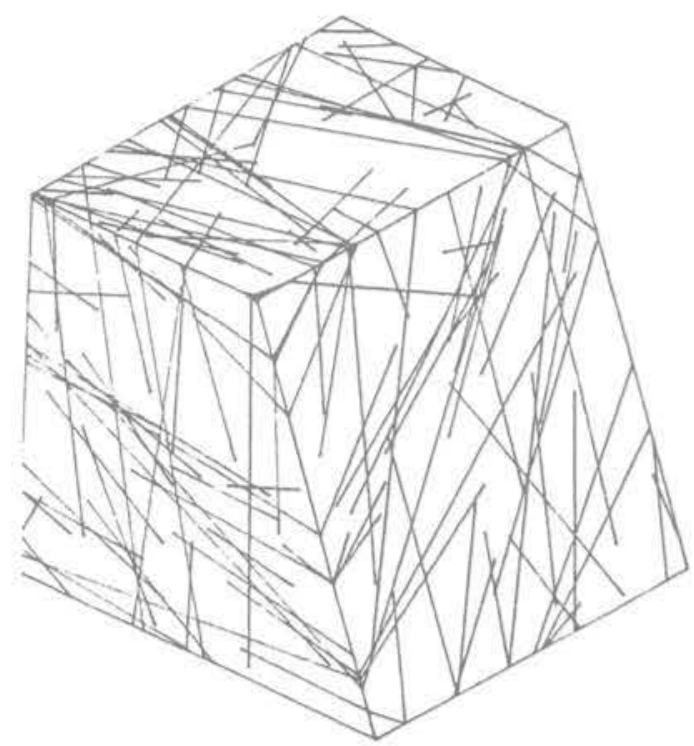

Fig. 4. - Réseau des discontinuités de degré 2 de connectivité. Fig. 4. - Discontinuity network with two degree connectivity.

L'étude de la connectivité pour la blocométrie de massifs rocheux consiste en une étude des traces des intersections sur chaque discontinuité et est réalisée dans un système local de coordonnées installé sur chaque discontinuité. La procédure est semblable à celle expliquée ci-dessus si une discontinuité est considérée comme un segment d'intersection et une trace d'intersection comme un point d'intersection. Une discontinuité sur laquelle il n'existe pas un réseau de traces d'intersection doit être éliminée par l'algorithme (4). Le résultat de cette étude pour le système de discontinuités de la figure 2 est montré dans la figure 5 .

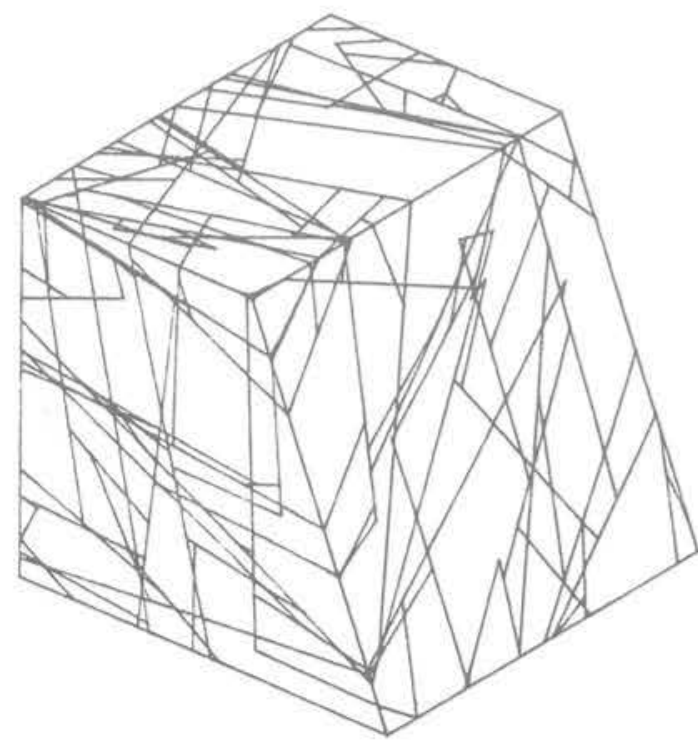

Fig. 5. - Réseau des discontinuités pour la blocométrie. Fig. 5. - Discontinuity network for block size analysis.
L'algorithme (4) concerne l'étude de la connectivité de discontinuités individuelles, mais ne concerne pas celle de réseaux de discontinuités. Dans un système de fissures, il existe souvent des réseaux isolés (locaux) par rapport au réseau global connecté à la limite du domaine intéressé. Par exemple dans la figure 3 , le réseau de fissures $\mathrm{N}^{\circ} 15,16,17,18$ est un réseau local. Au terme de la théorie de graphes, il concerne la connexité d'un graphe et le nombre de réseaux correspond au nombre de composantes connexes d'un graphe. Dans un réseau individuel, il peut exister des fissures ( $N^{\circ} 10$, etc dans la figure 3 ) et des intersections dont la suppression génère des nouveaux réseaux. Ils correspondent aux points d'articulation et aux isthmes dans la théorie de graphes. La recherche du réseau global, des points d'articulation et des isthmes peut être réalisée selon les algorithmes standards.

\section{IDENTIFICATION GLOBALE DE SOMMETS, D'ARÊTES ET DE FACETTES}

L'identification globale des sommets, des arêtes et des facettes est réalisée premièrement dans un système local de coordonnées installé sur chaque discontinuité. La figure $6 \mathrm{~A}$. montre une discontinuité circulaire et les traces des intersections avec d'autres discontinuités. Un sommet $V$ est un point d'intersection entre deux traces et donc est un point d'intersection entre trois discontinuités $\mathrm{Fi}, \mathrm{Fj}$, Fk qui définissent l'association du sommet $\mathrm{V}$, notée :

$$
\Gamma(\mathrm{V})=(\mathrm{Fi}, \mathrm{Fj}, \mathrm{Fk})
$$

Une arête $E$ est un segment entre deux sommets $V_{i}$ et $V_{m}$ qui définissent l'association de l'arête $\mathrm{E}$, notée :

$$
\Gamma(\mathrm{E})=(\mathrm{Vi}, \mathrm{Vm})
$$

C'est donc un segment sur la ligne d'intersection entre deux discontinuités. Une facette est définie comme un cycle élémentaire orienté dont la frontière est constituée d'arêtes orientées (fig. 6B.).

Du fait qu'un sommet global est une intersection entre trois discontinuités, il peut apparaître dans différentes discontinuités. De même, du fait qu'une arête globale est une intersection entre deux discontinuités, elle peut aussi apparaître dans différentes discontinuités. La transformation vers le système global doit considérer ce phénomène, le même sommet ou la même arête doit avoir un même numéro global unique quand il est transféré de différents systèmes locaux.

Si nous notons $V$ l'ensemble des sommets obtenus dans la procédure d'identification des sommets globaux et $\Gamma(W)$ l'ensemble des associations de sommets identifiés, alors pour un sommet $\mathrm{V}$, s'il est déjà identifié dans le système local sur l'une de ses trois fissures, $\Gamma(V)$ est déjà contenu dans $\Gamma(W)$ :

$$
\Gamma(\mathrm{V}) \cap \Gamma(\mathrm{V})=\Gamma(\mathrm{V})
$$



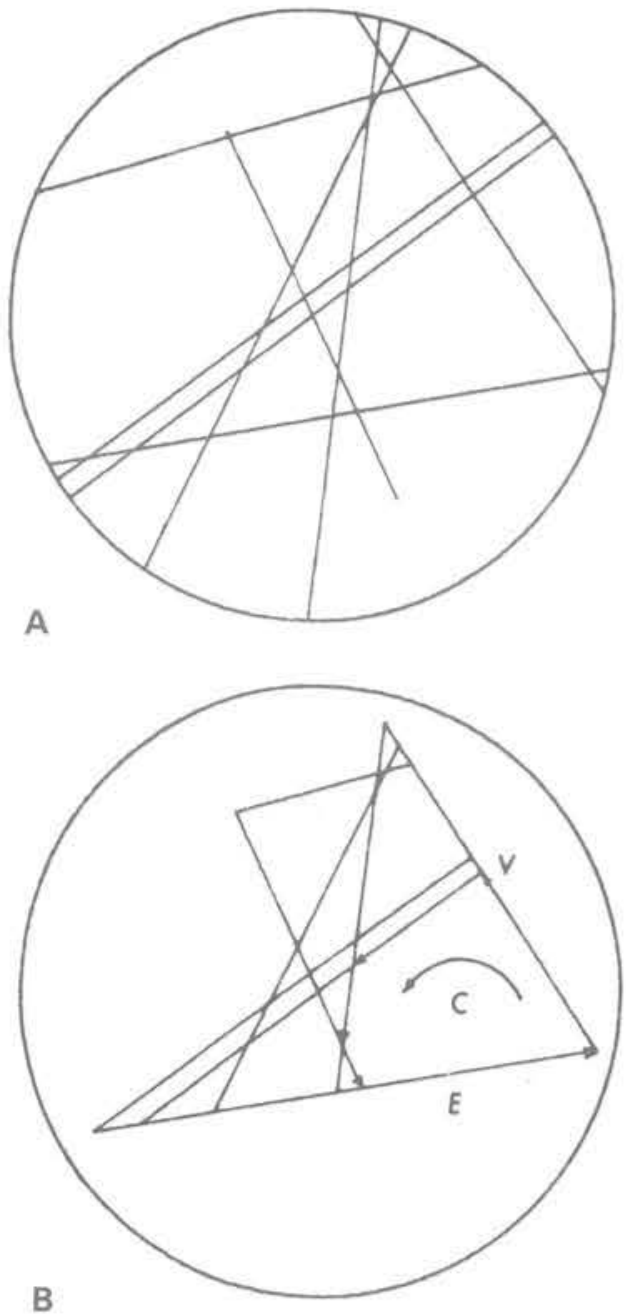

Fig. 6. - A. Traces des intersections sur une discontinuité de type disque.

B. Sommets, arêtes et cycles sur la discontinuité.

Fig. 6. A. Intersection traces on a dice shaped discontinuity. $B$. Vertices, edges and cycles an a discontinuity.

S'il est nouveau, on a :

$$
\Gamma(\mathrm{V}) \cap \Gamma(\mathrm{V})=\phi
$$

et on doit l'ajouter dans $\mathrm{V}$

$$
\begin{aligned}
& \mathbb{V} \leftarrow \mathbb{V} \cup V \\
& \Gamma(\mathbb{V})-\Gamma(\mathbb{V}) \cup \Gamma(\mathrm{V})
\end{aligned}
$$

De la même façon, si nous notons $\mathbb{E}$ l'ensemble des arêtes identifiées et $\Gamma(\mathbb{E})$ l'ensemble des associations des arêtes identifiées, alors pour une arête E, si elle est déjà identifiée, $\Gamma(E)$ est déjà contenu dans $\Gamma(\mathbb{E})$ :

$$
\Gamma(\mathbb{E}) \cap \Gamma(E)=\Gamma(E)
$$

Si elle est nouvelle, on a :

$$
\Gamma(\mathbb{E}) \cap \Gamma(E)=\phi
$$

et on doit l'ajouter dans (E)

$$
\begin{aligned}
& \mathbb{E}-\mathbb{E} \cup \mathbb{E} \\
& \Gamma(\mathbb{E})-\Gamma(\mathbb{E}) \cup \Gamma(E)
\end{aligned}
$$

Une facette, notée $\mathrm{C}$, étant présente sur une seule discontinuité, le problème précédent n'existe pas. La méthode d'identification d'un cycle élémentaire est identique à celle d'identification d'un bloc bidimensionnel.

\section{IDENTIFICATION DES BLOCS DISCRETS}

L'algorithme pour identifier un bloc discret intersecté par des discontinuités se base sur l'homomorphisme du bloc. Un bloc polyédrique est homomorphique ou identique au sens topologique à une sphère s'il peut s'identifier à celle-ci par une déformation continue, (LIN D et al., 1987). Le nombre de sommets, d'arêtes et de facettes de cette classe de blocs suit la formule d'EULER

$$
V-E+C=2
$$

et notre étude se limite à cette classe de blocs.

Du point de vue de la connexion du domaine, les facettes d'un bloc homomorphique engendrent un domaine de simple connexion. Si nous notons la normale extérieure à la surface du bloc par $\vec{n}$, selon la formule géométrique, l'intégrale de $\vec{n}$ suivant la surface fermée du bloc est nulle,

$$
\iint_{S} \vec{n} \text { ds }=\sum_{k=1}^{N c} S k \cdot \vec{n} k=\overrightarrow{0}
$$

avec Nc nombre de facettes d'un bloc

$\vec{n} \mathrm{k}$ normale extérieure à la facette $\mathrm{k}$

Sk surface de la facette $k$

$\overrightarrow{0}$ vecteur nul.

Une facette d'un bloc peut être représentée par un polygone dans l'espace dont la surface orientée $S \cdot \vec{n}$ peut être évaluée pour calculer le moment du polygone, l'équation (13) peut être écrite:

$$
\frac{1}{2} \sum_{k=1}^{N c} \sum_{i=k}^{m k} \vec{r}{ }^{i o} \times \vec{E}_{i}=\overrightarrow{0}
$$

avec $\mathrm{mk}$ nombre d'arêtes de la facette $\mathrm{k}$

$\overrightarrow{\mathrm{E}} \mathrm{i}$ arête orientée $\mathrm{i}$

$\vec{r}$ io un point de référence sur l'arête $\mathrm{i}$

$x$ symbole de produit vectoriel.

Pour un polyèdre, une arête correspond à deux facettes voisines, si nous notons les orientations d'une arête sur deux facettes voisines $j$ et $k$ par $\vec{E}$ et $\vec{E}^{k}$, l'équation (14) est identique à l'équation suivante :

$$
\begin{aligned}
& \frac{1}{2} \sum_{i=1}^{N a}\left[\left(\vec{r}{ }^{i 0} \times \vec{E}_{i}^{j}\right)+\left(\vec{r} i 0 \times \vec{E} i^{k}\right)\right] \\
& =\frac{1}{2} \sum_{i=1}^{N a}\left[\vec{r}^{i 0} \times\left(\vec{E} i^{j}+\vec{E} i^{k}\right)\right]=\overrightarrow{0}
\end{aligned}
$$

avec Na nombre d'arêtes d'un bloc. 
Du fait que $\mathrm{Na}$ points de référence $\overrightarrow{\mathrm{r}}^{\text {io }}$ sont non nuls et indépendants, on peut en déduire la relation suivante :

$$
\vec{E}_{i}+\vec{E}_{i}^{k}=\overrightarrow{0}
$$

L'équation (16) montre qu'une arête commune à deux facettes d'un bloc y présente des orientations opposées (fig. 7). C'est une propriété géométrique importante d'un bloc pour l'orienter et l'identifier et elle constitue la base de l'algorithme informatique de notre étude.

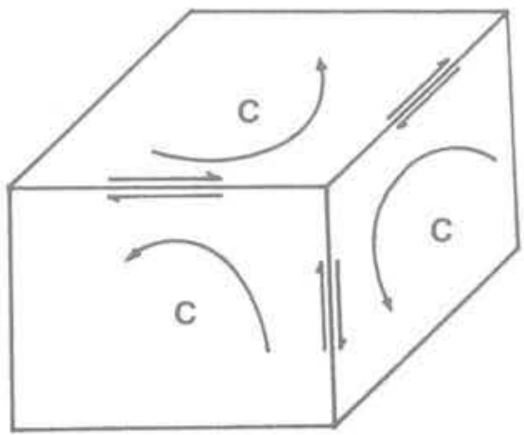

Fig. 7. - Orientation de l'arête commune à deux facettes. Fig. 7. - Orientation of an edge common to two faces.

Du fait qu'une arête possède une orientation opposée sur deux facettes voisines, elle s'élimine sur cellesci. Une arête n'appartient toujours qu'à deux facettes voisines sur un bloc, donc toutes les arêtes d'un bloc s'éliminent. En terme topologique, cela signifie que la frontière topologique de la surface d'un bloc est vide (LIN et al., 1987). Si nous notons l'ensemble des arêtes d'une facette par $\mathbb{C}$, alors nous pouvons définir un ensemble d'arêtes de $k$ facettes comme:

$$
\left\{\begin{array}{l}
\mathbb{D} k=\left[\mathbb{D} k-1-\left(\mathbb{C} k \cap \bigcup_{i=1}^{k-1} \mathbb{C} i\right)\right] \\
\cup\left(\mathbb{C} k-\bigcup_{i=1}^{k-1} \mathbb{C} i\right) ; k \geq 2 \\
\mathbb{D} 1=\mathbb{C} 1
\end{array}\right.
$$

Quand l'équation (17) est appliquée à toutes les facettes, i.e. quand $\mathrm{k}=\mathrm{Nf}$ (nombre de facettes d'un bloc), IDk sera un ensemble vide, car toutes les arêtes se sont éliminées. L'équation (17) est écrite sous la forme itérative pour la réalisation informatique et elle sert comme un critère de la fermeture du bloc. Au cours d'identification d'un bloc, si nous sommes arrivés à une facette $\mathrm{k}$ telle que $\mathbb{D k}=\phi$, alors le bloc est identifié et la valeur de $\mathrm{k}$ donne le nombre de facettes du bloc.

En se basant sur les équations (16) et (17), l'identification d'un bloc tridimensionnel cormmence par une facette. Une facette intérieure peut constituer la facette commune à deux blocs voisins correspondant à deux normales opposées (fig. 8). Par contre, une facette sur la surface du domaine ne correspond qu'à un bloc réel, l'autre est un bloc virtuel constitué par toutes les facettes sur la surface. La différence entre eux est que

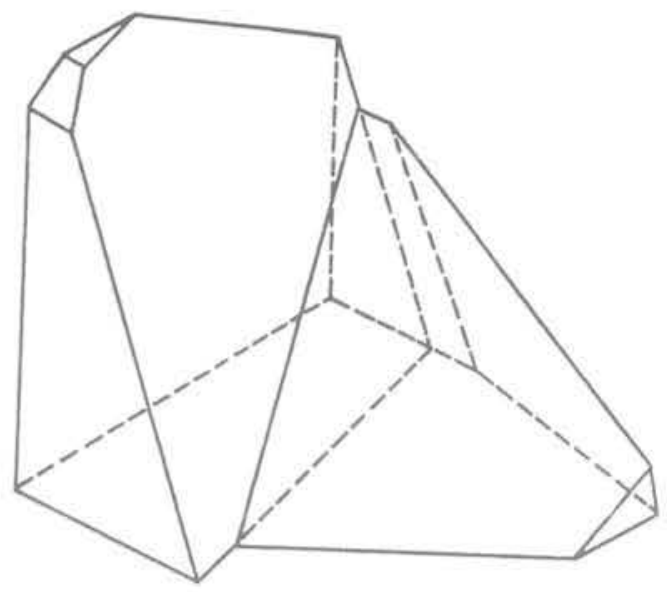

Fig. 8. - Deux blocs voisins correspondant à une facette intérieure.

Fig. 8. - Two neighbour blocks corresponding to an interior face.

le bloc virtuel a un volume négatif et ne sera pas compté dans la blocométrie.

Dans le processus d'identification, une facette est orientée pour que la normale à la facette soit toujours vers l'extérieur du bloc. Ceci peut être réalisé en considérant la propriété de l'équation (16). Suivant l'équation (16), nous pouvons orienter une facette candidate en vérifiant l'orientation de ses arêtes par rapport à l'orientation des arêtes des facettes adjacentes identifiées. Par exemple, dans la figure 9, supposons que la facette en cours est $\mathrm{Ck}$, la normale à cette facette est $\vec{n} k$, nous voulons identifier la facette suivante du bloc liée à l'arête Ei. Il existe peut-être plusieurs facettes candidates, leurs orientations doivent être vérifiées et modifiées si nécessaire suivant leurs relations par rapport à celle de l'arête sur $\mathrm{Ck}$.

Soit $\mathrm{M}$ facettes candidates, nous notons les vecteurs normalisés de moments de ces facettes par $\vec{m} i(i=1$, M). Pour identifier la facette suivante, il convient d'établir un système de coordonnées $\mathrm{O}(\xi \eta \zeta)$ installé sur l'arête $\vec{E}_{\ell}$ de la facette $\mathrm{Ck}$. Les vecteurs de base $\left.\vec{e}_{\xi}, \vec{e}_{\eta}, \vec{e}_{\xi}\right)$ du système suivent les conditions suivantes:

$$
\begin{aligned}
& \vec{e}_{\xi} \times \vec{e}_{\eta}=\vec{e}_{\zeta} \\
& \vec{e}_{\zeta} \cdot \vec{E}_{\ell}=\left|\vec{E}_{\ell}\right|
\end{aligned}
$$

Le problème de déterminer la facette suivante devient celui de la détermination du vecteur $\overrightarrow{\mathrm{m}}$ l le plus à gauche par rapport à $\vec{n} \mathrm{k}$ dans le plan $(\xi \eta)$ du système comme il est montré dans la figure $9 \mathrm{C}$ et l'étude suivante se limite dans ce plan, c'est-à-dire, les vecteurs $\vec{m}$ et $\vec{n}$ sont considérés comme bidimensionnels.

Nous notons le nombre de facettes candidates à gauche du vecteur en cours $\vec{n} k$ par Ml, alors nous avons 


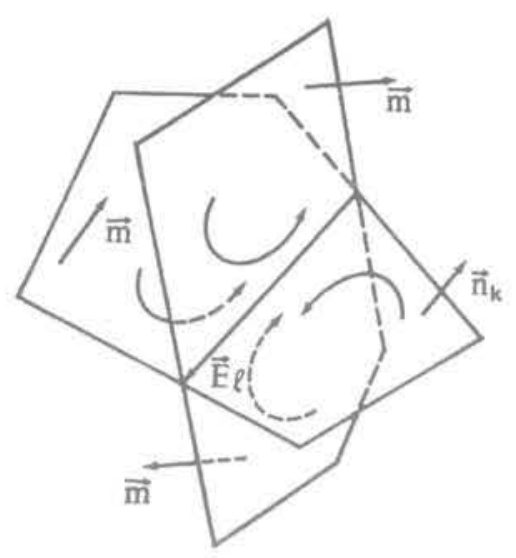

A

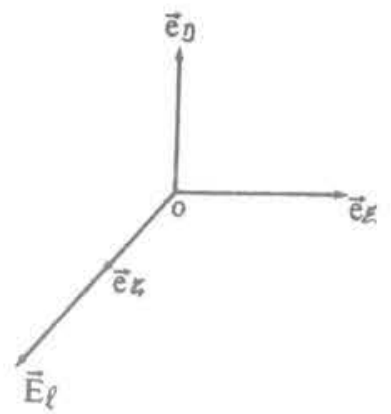

B

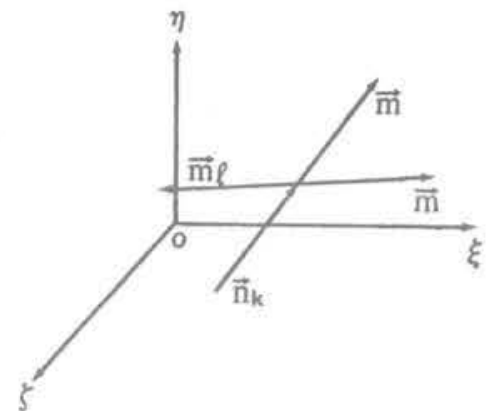

C

Fig. 9. - Identification de la facette la plus à gauche.

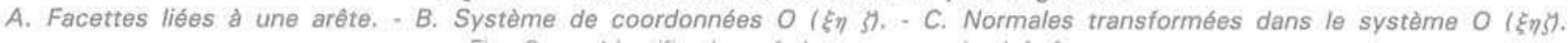
Fig. 9. - Identification of the most on the left face.

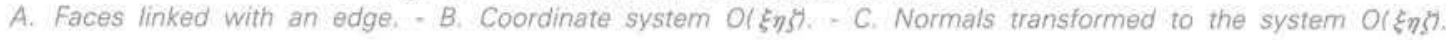

$\mathrm{Ml} \leq \mathrm{M}$. Si $\mathrm{Ml}>0$, le vecteur le plus à gauche $\overrightarrow{\mathrm{m} l}$ sera déterminé par:

$$
\begin{aligned}
& |\vec{n} k-\overrightarrow{m l}|=\max \\
& \{|\vec{n} k-\vec{m} l|, i=1, M l\}
\end{aligned}
$$

Si $\mathrm{Ml}=0$, alors $\overrightarrow{\mathrm{m} l}$ sera déterminé par :

$$
\begin{aligned}
& |\vec{n} k-\vec{m} l|=\min \\
& \{|\vec{n} k-\vec{m} l|, i=1, M\}
\end{aligned}
$$

Une fois que $\overrightarrow{\mathrm{m}} \mathrm{l}$ est identifié, nous sommes arrivés à une nouvelle facette. Le problème suivant est de vérifier la fermeture du bloc pour décider s'il faut continuer d'identifier les facettes suivantes ou non. Cela peut être réalisé par l'équation (17). Si l'ensemble $\mathbb{D}$ est vide, le bloc est identifié, si non, nous choisissons une arête dans $\mathbb{D}$, normalement la première, comme l'arête en cours, nous cherchons les facettes candidates liées à cette arête et nous répétons les procédures ci-dessus.

La surface d'un cycle orienté (facette) peut être évaluée pour calculer son moment et est égale à la moitié de celui-ci. Supposons qu'un cycle soit constitué de $\mathrm{m}$ arêtes et également de $\mathrm{m}$ sommets. Si une facette est identifiée par la méthode présentée, l'orientation des arêtes est définie dans le sens trigonométrique, la surface de l'arête s'écrit:

$$
S=\frac{1}{2} \sum_{i=1}^{m} \vec{r} i 0 \times \vec{E} j
$$

où $\vec{E}_{i}$ est une arête orientée, $\vec{r}^{\text {io }}$ est un point de référence et peut être choisi comme un point quelconque sur la ligne de l'arête $\overrightarrow{\mathrm{E}}_{\mathrm{i}}$.

Le volume d'un bloc de $\mathrm{n}$ facettes peut être calculé par la surface de ses facettes et la normale extérieure à chaque facette.

$$
V=(1 / 3) \sum_{i=1}^{n}(\vec{r} \circ, \vec{n} i) \cdot S i
$$

avec $\vec{n} \mathrm{i}$ normale à la facette $\mathrm{i}$

Si surface de la facette i

$\vec{r}$ io point de référence sur facette $i$

Le point de référence peut être choisi comme un point quelconque sur le plan de la facette i, par exemple un de ses sommets.

La méthode présentée ci-dessus pour identifier un bloc ne fait pas l'hypothèse de la convexité du bloc. Un bloc concave peut être identifié de la même manière qu'un bloc convexe, mais nous avons supposé que les blocs sont homomorphiques en terme de topologie. Cela sera généralement satisfait si une étude de la connectivité des discontinuités et des traces des intersections sur chaque discontinuité est faite et si les réseaux locaux de discontinuités sont éliminés.

\section{BLOCOMÉTRIE DE MASSIFS ROCHEUX}

Nous allons discuter la distribution de taille de blocs d'un massif rocheux. La taille d'un bloc peut être représentée par son volume ou plus simplement par son diamètre équivalent. Dans ce texte, nous utilisons la racine cubique du volume pour présenter la taille moyenne d'un bloc. Pour un massif rocheux, la distribution de taille de blocs $\mathrm{F}(\mathrm{x})$ présente certains caractères de la structure. Par exemple, on peut mesurer le pourcentage de nombre de blocs trouvés dans un intervalle de taille donnée. La figure 10 montre la distribution de taille et l'histogramme de fréquence du système de blocs dans la figure 5 .

L'inconvénient de la présentation de $\mathrm{F}(\mathrm{x})$ est qu'elle insiste seulement sur l'importance en nombre, et ne montre pas clairement l'importance de la taille ellemême. Dans un massif rocheux, l'intersection entre 


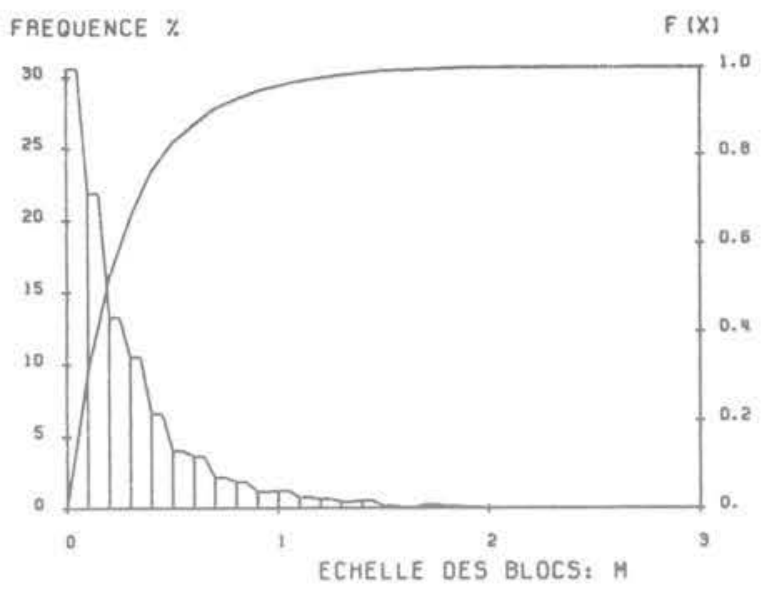

Fig. 10. - Distribution de taille des blocs. Fig. 10. - Block size distribution.

des discontinuités aléatoires peut engendrer un très grand nombre de blocs de petite taille, le volume de ces petits blocs pouvant être très faible. Leur influence sur la stabilité ou la fragmentation d'un massif rocheux ne sera pas aussi importante qu'on pourrait le penser au vu de leur pourcentage en nombre. Ainsi nous utilisons une autre méthode de représentation de la blocométrie, appelée distribution de taille pondérée $\mathrm{G}(\mathrm{x})$ définie comme le pourcentage du volume des blocs de diamètre inférieur à une valeur $\mathrm{x}$, sur le volume total des blocs du système.

$$
G(x)=\frac{1}{\int_{0}^{\infty} u^{3} f(u) d u} \int_{0}^{x} u_{3} f(u) d u
$$

Cette distribution et l'histogramme de fréquence du système des blocs de la figure 5 sont présentés dans la figure 11. On peut voir la différence importante avec la figure 10.

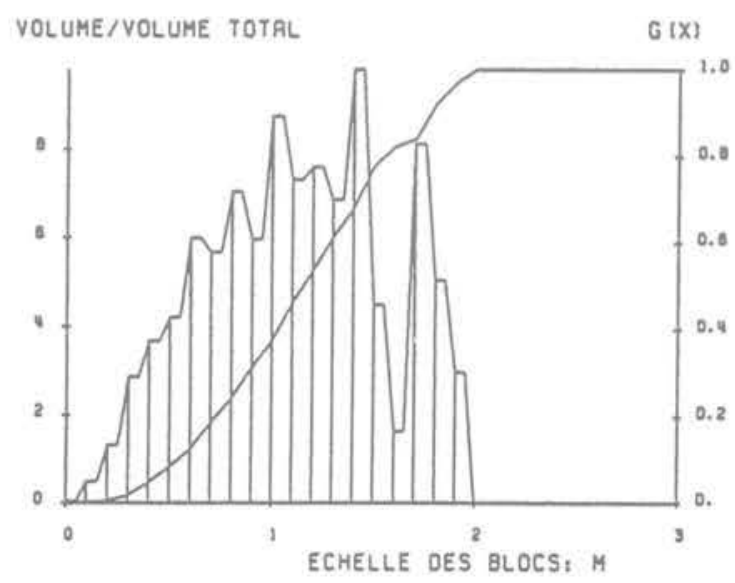

Fig. 11. - Distribution de taille pondérée.

Fig. 11. - Weighted block size distribution,

Une autre propriété géométrique importante des blocs pour l'analyse de la stabilité et l'évaluation de la fragmentation est la distribution de l'orientation des blocs. L'orientation d'un bloc est définie dans cet article par celle de l'axe principal maximum. La distribution sphérique de l'orientation des blocs peut être présentée par la projection stéréographique et le résultat du système de blocs de la figure 5 est présenté dans la figure 12 .

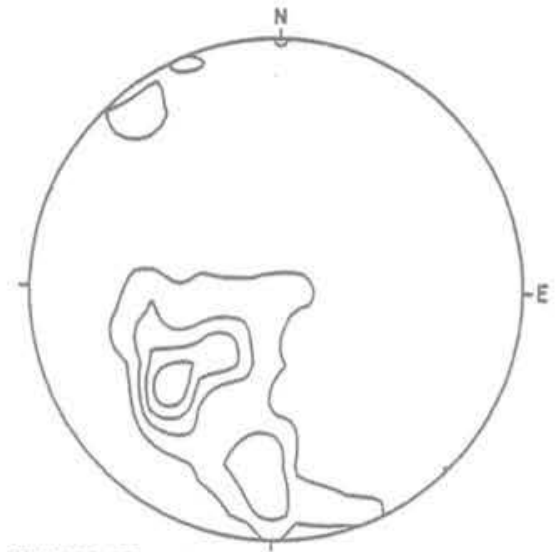

STEREO OE L'AXE MAX. DES GLOCS

Fig. 12. - Projection stéréographique de l'orientation des blocs.

Fig. 12. - Stereographic projection of block orientations.

\section{CONCLUSION}

La méthode présentée dans l'article pour identifier un bloc discret intersecté par des discontinuités utilise les méthodes simples de la géométrie, et ne se base pas ur l'hypothèse de la convexité des blocs. Les blocs concaves peuvent être identifiés de la même façon que les blocs convexes. Les études de la connectivité des discontinuités nous permettent de fournir les modèles géométriques nécessaires à l'analyse des écoulements, à l'analyse mécanique ou à l'analyse couplée hydromécanique. L'évaluation granulométrique donne certaines informations sur la structure d'un massif rocheux et peut être appliquée à l'analyse de la stabilité et l'étude de la fragmentation.

\section{BIBLIOGRAPHIE}

ANDERSSON J., DVERSTORP B. (1987), Conditional simulation of fluid flow in three dimensional networks of discrete fractures. Water Resources Research, vol. 23, n० $10,1876-1886$.

BAECHER G.B. (1983), Statistical analysis of rock mass fracturing. Mathe. Geol., vol. 15 (2), 329-348.

BILLAUX D. (1989), Three dimensional statistical modelling of a fractured rock mass. An example from the Fanay Augères Mine. Int. J. Rock Mech. Min. Sci. \& Geomech. Abstr., vol. 26, n०3/4, pp. 281-299.

CUNDALL P.A. (1971), A computer model for stimulating progressive large scale movements in blocky rock systems. Proc. Int. Symp. Rock Fracture, ISRM, Nancy.

CUNDALL P.A. (1988), Formulation of a three dimensional distinct element model. Int. J. Rock. Mech. Min. Sci. \& Geomech. Abstr., vol. 25, 107-125. 
LIN D., FAIRHURST C., STARFIELD A.M. (1987), Geometrical identification of three dimensional rock block systems using topological techniques. Int. J. Rock. Mech. Min. Sci. \& Geomech. Abstr., vol. 24, 331-338.

LONG J.C.S., GILMOUR P., WITHERSPOON P.A. (1985), A model for steady fluid flow in random three dimensional networks of disc-shaped fractures. Water resources research, vol. $21, n^{\circ} 8$, pp. 1105-1115.

MAHTAB M.A. (1972), Analysis of fracture orientations for input to structural models of discontinuous rock. Bureau of Mines Report of Investigations, US Department of the Interior.
PAHL P.J. (1981), Estimating the mean length of discontinuity traces. Inter. Jour. Rock Mech. Min. Sci. \& Geomech. Abstr., vol. 18, pp. 229-234.

PRIEST S.D., HUDSON J.A. (1981), Estimation of discontinuity spacing and trace length using scanline surveys. Inter. Jour. Rock Mech. Min. Sci. \& Geomech. Abstr., vol. 18, pp. 183-187.

VINCHES M. (1988), Application combinée de l'analyse structurale, de la géostatistique et de la théorie des blocs clés à l'étude des problèmes de stabilité et de confortement des travaux miniers. Rev. Franç. Géotech., n 45, pp. 59-70.

WARBURTON P.M. (1980), A stereological interpretation of joint trace data. Int. J. Rock. Mech. Min. Sci. \& Geomech. Abstr., vol. 17, 181-190. 